\title{
Microestruturas de pêlos de pequenos mamíferos não-voadores: chave para identificação de espécies de agroecossistemas do estado de São Paulo, Brasil
}

\author{
Paula Sanches Martin ${ }^{1}$, Carla Gheler-Costa ${ }^{1,2}$ \& Luciano Martins Verdade ${ }^{1}$ \\ ${ }^{1}$ Laboratório de Ecologia Animal, \\ Escola Superior de Agricultura “Luiz de Queiroz” - ESALQ, \\ Universidade de São Paulo - USP, \\ CP 09, CEP 13418-900, Piracicaba, SP, Brasil \\ ${ }^{2}$ Autor para correspondência: Carla Gheler-Costa, e-mail: cgcosta@esalq.usp.br
}

MARTIN, P.S., GHELER-COSTA, C. \& VERDADE, L.M. 2009. Microstructures of the hair of non-volant small mammals: key to the identification of species from agroecosystems of the State of São Paulo, Brazil. Biota Neotrop. 9(1): http://www.biotaneotropica.org.br/v9n1/en/abstract?identification-key+bn01509012009.

Abstract: Hair microstructure characteristics have been used for species identification in taxonomic, ecological, paleontological, archeological and forensic research. This study aims to describe the hair structure from small mammals commonly found in agroecosystems of the São Paulo State. The hairs were collected from the dorsal region of previously identified specimens collected in agricultural areas (sugar-cane plantations), pasture areas, silviculture areas (eucalyptus plantation) and fragments of native vegetation (semideciduos Atlantic Forest and Cerrado). Optic microscope was not effective for describing the form and the border of scales, which were described using scanning electronic microscope. A dichotomic key of identification is presented for 11 species of non-volant small mammals (seven rodents and four marsupials).

Keywords: cuticle, guard-hairs, medulla, small mammals, tricology.

MARTIN, P.S., GHELER-COSTA, C. \& VERDADE, L.M. 2009. Microestruturas de pêlos de pequenos mamíferos não-voadores: chave para identificação de espécies de agroecossistemas do estado de São Paulo, Brasil. Biota Neotrop. 9(1): http://www.biotaneotropica.org.br/v9n1/pt/abstract?identificationkey+bn01509012009.

Resumo: Características microestruturais dos pêlos vêm sendo usadas para identificação de espécies em pesquisas taxonômicas, ecológicas, paleontológicas, arqueológicas e forenses. Este estudo tem como objetivo a descrição da estrutura dos pêlos de algumas espécies de pequenos mamíferos não-voadores comumente encontrados em agroecossistemas do Estado de São Paulo. Os pêlos foram coletados do dorso de espécimes previamente identificados coletados em áreas de agricultura (canavial), pastagens, silvicultura (plantio de eucalipto) e fragmentos de vegetação nativa (floresta semidecídua e cerrado). O microscópio óptico apresentou limitações para a descrição do formato e borda das escamas, os quais foram descritos através do uso de microscópio eletrônico de varredura. Uma chave de identificação dicotômica é apresentada para 11 espécies de pequenos mamíferos não-voadores (sete roedores e quatro marsupiais).

Palavras-chave: cutícula, medula, pêlos-guardas, pequenos mamíferos, tricologia. 


\section{Introdução}

Desde o início do século passado, pesquisadores já tinham conhecimento de que a superfície externa dos pêlos apresenta estrutura e forma variáveis. Hausman (1920) deu início ao estudo da estrutura dos pêlos como caráter diferencial entre as espécies de mamíferos e publicou uma série de trabalhos propondo nomenclatura, classificações e técnicas, que serviram de subsídio para pesquisas na área, que passou a ser chamada de tricologia. Posteriormente vários trabalhos contribuíram para seu desenvolvimento (Carter \& Dilworth 1971, Weingart 1973, Keller 1980, Teerink 1991, Quadros 1998, Quadros \& Monteiro-Filho 1998a, 1998b, Broeck et al. 2000, Chernova 2002, Meyer et al. 2000, 2002, Porta \& Llanesa 2001, Hoey et al. 2004). Atualmente a tricologia tem sido utilizada como base para estudos aplicados em paleontologia (Meng \& Wiss, 1997), sistemática (Mathiak 1938, Nason 1948, Mayer 1952, Keller 1980, Teerink 1991, Broeck et al. 2000, Anderson 2001, Porta \& Llanesa 2001, Andruskiw 2003, Ingberman \& Monteiro-Filho 2006), ecologia (Hilton \& Kutscha 1978, Cypher 1993a, 1993b, Quadros 1998) e ciência forense (Houck 2002).

Teerink (1991) sugere que os pêlos sejam divididos em duas grandes categorias: pêlos-guardas ("overhair") e subpêlos ("underhair"). Os pêlos-guardas são mais longos, mais numerosos, grossos, sobressaindo-se (Brunner \& Coman 1974) e produzindo o padrão geral de coloração da pelagem (Quadros 1998), apresentando função mecanorreceptora e dissimuladora no ambiente. Os subpêlos são mais curtos, mais finos, ondulados e numerosos (Brunner \& Coman 1974), contribuindo para a termorregulação e proteção (Mathiak 1938).

A cutícula, a medula e o córtex dos pêlos-guardas apresentam padrões morfológicos que, combinados entre si, conferem a uma determinada espécie características únicas e definidas, que podem ser utilizadas em identificação taxonômica (Hausman 1920, Keller 1980, Meyer 2002, Ingberman \& Monteiro-Filho 2006, Quadro \& Monteiro-Filho 2006a) e estudos sobre ecologia alimentar de carnívoros (Mathiak 1938, Nason 1948, Mayer 1952, Teerink 1991, Fernández \& Rossi 1998, Quadros 1998, Andruskiw 2003).

Estudos sobre pêlos de mamíferos sul-americanos são escassos (Piantanida \& Petriella 1976, Chehébar \& Martín 1989, Muller 1989, Quadros \& Monteiro-Filho 1998a, 1998b, 2006a, 2006b, Fernandez \& Rossi 1998, Vázquez et al 2000, Ingberman \& Monteiro-Filho 2006), sendo a maioria dos trabalhos de tricologia referentes a mamíferos da América do Norte (Mathiak 1938, Nason 1948, Mayer 1952, Hilton \& Kutscha 1978, Andruskiw 2003), Europa (Hausman 1920, Keller 1980, Teerink 1991, Broeck et al. 2000, Porta \& Llanesa 2001, González-Esteban et al. 2006, Marinis \& Asprea 2006), e Oceania (Brunner \& Coman 1974).

Os trabalhos referentes à tricologia no Brasil foram desenvolvidos com amostras provenientes de animais vivos e/ou taxidermizados (Muller 1989, Quadros \& Monteiro-Filho 1998a, 1998b, 2006a, 2006b, Ingberman \& Monteiro-Filho 2006). Estes trabalhos foram aplicados para identificação de presas e/ou predadores de áreas florestadas e conservadas. Estudos referentes a pequenos mamíferos em agroecossistemas são insipientes (Barros-Batesti et al. 2000, Bonvicino et al. 2002, Bush et al. 2001, Gheler-Costa et al. 2002, Gheler-Costa 2006), se considerarmos agroecossistema como um conjunto que compreende ecossistemas naturais e ambientes modificados pelo homem (Odum et al. 1988). A grande parte dos estudos de ecologia de pequenos foram realizados em áreas conservadas ou com porcentagem de vegetação natural acima de 20\% (Castro \& Fernandez 2004, Fonseca \& Robinson 1990, Pardini 2004, Pardini et al. 2005, Pires et al. 2002, Pires et al. 2005).

O presente estudo visa ampliar o conhecimento sobre a tricologia de mamíferos de pequeno porte não voadores que habitam agroecos- sistemas do Estado de São Paulo, por meio da elaboração de uma chave de identificação, baseada na microestrutura dos pêlos, para servir como subsidio a pesquisas relacionadas à dieta de carnívoros em agroecossistemas.

\section{Material e Métodos}

\section{1. Áreas de estudo}

As amostras de pêlos do presente estudo são provenientes de espécimes capturados nas seguintes áreas de estudo: Bacia do rio

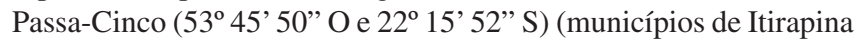
e Ipeúna), Fazendas Ibiti (49 $16^{\circ} 52^{\prime \prime} \mathrm{O}$ e $24^{\circ}$ 9' 50” S) (município de Itararé) e Santa Isabel (49 5' 54" O e 24 10'20” S) (município de Itapeva). A bacia do rio Passa-Cinco possui 52.757,60 ha, que compõe juntamente com outras quatro sub-bacias a Bacia do Rio Corumbataí, na região central do Estado de São Paulo. A região apresenta um mosaico de ambientes, caracterizando-se como um agroecossistema, sendo a matriz composta por pastagens $(51,72 \%)$, além de áreas com plantação de cana-de-açúcar (14,1\%), florestas plantadas de Eucalipto $(10,75 \%)$ e fragmentos florestais nativos $(15,67 \%)$, tanto de cerrado, como de floresta estacional semidecidual (Koffler 1993, Valente 2001).

As fazendas Ibiti (11.300 ha) e Santa Isabel (5840 ha) destinam-se à produção silvicultural com plantios de eucalipto e localizam-se na região sudoeste do Estado de São Paulo. A região caracteriza-se por ser uma zona de transição entre os seguintes sistemas fisionômicoecológicos: floresta estacional semidecidual, floresta ombrófila densa montana e cerrado.

\section{Coleta de dados}

Na Bacia do Passa-Cinco, utilizaram-se armadilhas do tipo Sherman distribuídas em 16 sítios de amostragem, igualmente distribuídos entre fragmentos de floresta semidecídua, plantios de eucalipto, pastagens e canaviais (Gheler-Costa 2006). Nas Fazendas Ibiti e Santa Isabel, utilizaram-se armadilhas de interceptação e queda (pitfall) distribuídas em 9 sítios de amostragem, sendo 6 áreas de eucalipto (borda e interior), 2 fragmentos de floresta semidecídua e 1 fragmento de cerrado.

Os pêlos foram coletados na região de intersecção da linha mediana com a linha escapular no dorso de espécimes vivos. Um pequeno tufo de pêlos foi arrancado manualmente para a conservação do bulbo e ápice dos pêlos. As amostras foram então armazenadas em tubos plásticos identificados (Quadros \& Monteiro-Filho 2006a). Os espécimes foram identificados através de análises morfológicas de crânios depositados no Museu de Zoologia da Universidade de São Paulo (MZUSP).

Foram analisados os pêlos de 11 espécies, quatro marsupiais e sete roedores (Tabela 1). Apenas as características da cutícula e da medula dos pêlos-guardas foram descritas, por apresentarem similaridade para indivíduos de uma mesma espécie e diferenças entre as espécies. A nomenclatura utilizada foi descrita por Quadros \& Monteiro-Filho (2006b). Para uma descrição mais detalhada do formato das escamas foi necessária uma combinação entre a nomenclatura proposta por Hausman (1920) e Quadros \& Monteiro-Filho (2006b), por descreverem padrões de pêlos de espécies brasileiras.

Foram utilizados 10 pêlos de quatro indivíduos diferentes para cada espécie coletada. A limpeza dos pêlos foi realizada em álcool e a secagem em papel absorvente. Na obtenção de impressões cuticulares, lâminas foram montadas através da adição de uma fina camada de esmalte incolor para unhas. Após secagem de 20 minutos, as lâminas com os pêlos foram colocados e prensados entre dois pedaços de madeira em uma pequena morsa. Após a secagem total do esmalte, 
Microestrutura de pêlos de pequenos mamíferos

Tabela 1. Tipo de ambiente e local de coleta das espécies de pequenos mamíferos não-voadores analisados no presente estudo.

Table 1. Type of environment and collection locality of the non-volant small mammals analyzed in this study.

\begin{tabular}{|c|c|c|c|c|}
\hline Ordem & Família & Espécie & Tipo de ambiente & Local \\
\hline \multirow[t]{4}{*}{ Didelphimorphia } & Didelphidae & Didelphis albiventris (Lund, 1840) & $\begin{array}{l}\text { Fragmento de Floresta } \\
\text { Semidecídua e Plantio } \\
\text { de Eucalipto }\end{array}$ & $\begin{array}{l}\text { Bacia do Rio Passa-Cinco, } \\
\text { Itapeva e Itararé, SP }\end{array}$ \\
\hline & & Monodelphis americana (Muller, 1776) & $\begin{array}{l}\text { Fragmento de Floresta } \\
\text { Semidecídua }\end{array}$ & Bacia do Rio Passa-Cinco, SP \\
\hline & & Gracilinanus agilis (Burmeister, 1854) & $\begin{array}{l}\text { Fragmento de Floresta } \\
\text { Semidecídua e Plantio } \\
\text { de Eucalipto }\end{array}$ & $\begin{array}{l}\text { Bacia do Rio Passa-Cinco, } \\
\text { Itapeva e Itararé, SP }\end{array}$ \\
\hline & & Gracilinanus microtarsus (Wagner, 1842) & Plantio de Eucalipto & Itapeva e Itararé, SP \\
\hline \multirow[t]{7}{*}{ Rodentia } & Cricetidae & Rhipidomys mastacalis (Lund, 1840) & $\begin{array}{l}\text { Fragmento de Floresta } \\
\text { Semidecídua e Plantio } \\
\text { de Eucalipto }\end{array}$ & $\begin{array}{l}\text { Bacia do Rio Passa-Cinco, } \\
\text { Itapeva e Itararé, SP }\end{array}$ \\
\hline & & Oligoryzomys nigripes (Olfers, 1818) & $\begin{array}{l}\text { Fragmento de Floresta } \\
\text { Semidecídua, Plantio } \\
\text { de Eucalipto e Canavial }\end{array}$ & $\begin{array}{l}\text { Bacia do Rio Passa-Cinco, } \\
\text { Itapeva e Itararé, SP }\end{array}$ \\
\hline & & Calomys tener (Winge, 1837) & $\begin{array}{l}\text { Fragmento de Floresta } \\
\text { Semidecídua, Cerrado, } \\
\text { Plantio de Eucalipto, } \\
\text { Canavial e Pastagem }\end{array}$ & $\begin{array}{l}\text { Bacia do Rio Passa-Cinco, } \\
\text { Itapeva e Itararé, SP }\end{array}$ \\
\hline & & Akodon montensis (Thomas, 1913) & $\begin{array}{l}\text { Fragmento de Floresta } \\
\text { Semidecídua, Cerrado, } \\
\text { Canavial e Pastagem }\end{array}$ & $\begin{array}{l}\text { Bacia do Rio Passa-Cinco, } \\
\text { Itapeva e Itararé, SP }\end{array}$ \\
\hline & & Necromys lasiurus (Lund, 1841) & $\begin{array}{l}\text { Fragmento de Floresta } \\
\text { Semidecídua, Cerrado, } \\
\text { Plantio de Eucalipto e } \\
\text { Canavial }\end{array}$ & $\begin{array}{l}\text { Bacia do Rio Passa-Cinco, } \\
\text { Itapeva e Itararé, SP }\end{array}$ \\
\hline & & Bibimys labiosus (Winge, 1887) & Plantio de Eucalipto & Itapeva, SP \\
\hline & Echimyidae & $\begin{array}{l}\text { Kannabateomys amblyonix } \\
\text { (Wagner, 1845) }\end{array}$ & Plantio de Eucalipto & Itapeva, SP \\
\hline
\end{tabular}

os pêlos foram retirados da lâmina. Para a observação da medula, os pêlos foram expostos à água oxigenada cremosa 30 volumes por 80 minutos para descolorir, sendo em seguida lavados em água e secos com papel absorvente (Quadros \& Monteiro-Filho 2006a).

As lâminas foram preparadas utilizando-se do meio de montagem Entellan ${ }^{\circledR}$. Todas as amostras foram observadas em microscópio óptico modelo LEICA BM E, visualizadas no aumento 400x. Imagens de microscopia óptica foram obtidas a partir do software Image-pro plus, da media cybernetics - 1993-97 versão 3.0.00.00 para win 95/NT, câmera Sony CCD-IRIS modelo DXC-107-A, acoplada a microscópio de contraste de fase (LEICA BM E), aumento de 400x.

Para a observação da cutícula em microscópio eletrônico de varredura, as amostras passaram por um processo de deposição metálica para evitar um efeito conhecido como carga "charging", que é caracterizado pelo aparecimento de porções da superfície que se tornam intensamente brilhantes (Kitajima \& Leite 1999). Esta deposição foi efetuada em um sistema conhecido como "sputtering". As amostras foram montadas em "stubs" ou porta espécimes e inseridas no metalizador MED 010 da Balzer. Após este preparo as amostras foram analisadas em microscópio eletrônico de varredura DSM 900 da Zeiss. As observações da cutícula foram feitas nos aumentos 1000x, 2000x e 3000x.

\section{Resultados}

A medula apresentou-se de forma contínua e com maior variação entre as espécies na sua região distal. A medula foi identificada em todas as amostras ocupando toda a extensão do pêlo com arranjos celulares dispostos em uma ou mais fileiras, nas formas: a) isolada, quando se apresentou um espaço preenchido por córtex entre as células individualizando-as (Figura 1a); ou, b) anastomosada, quando havia fusões entre as células formando arranjos bem diversificados que delimitavam os espaços do córtex (Figuras 1b, c, d). Os formatos encontrados foram os mais variados: a) escalariforme, onde havia apenas uma única fileira de células aproximadamente retangulares e transversais em relação ao maior eixo do pêlo (Figura 1a); b) alveolar, quando a medula apresentou várias células fundidas em sua largura, delimitando espaços como cavidades ou alvéolos entre si (Figura 1b); c) listrada, quando as cavidades apresentaram-se fundidas e alinhadas em filas transversais em relação ao maior eixo do pêlo, formando barras transversais, delimitando espaços contínuos com grânulos de pigmento (Figura 1c); d) crivada, quando a medula apresentou mais que uma fileira de células, anastomosadas umas com as outras, circunscrevendo espaços com ausência de pigmento em seu interior (Figura 1d). O padrão medular de cada espécie está descrito na Tabela 2.

A maior variação do padrão cuticular foi verificada na região proximal do pêlo através de imagens provenientes dos microscópios ótico e de varredura. Os arranjos das escamas encontrados foram: a) imbricado, com escamas dispostas com a borda livre (distal) dirigida para o ápice do pêlo (Figura 2a, b e c, 3a, b, c, d) e b) pavimentosa, com escamas não apresentando bordas livres e sobreposição entre suas bordas adjacentes, assemelhando-se a azulejos na parede 

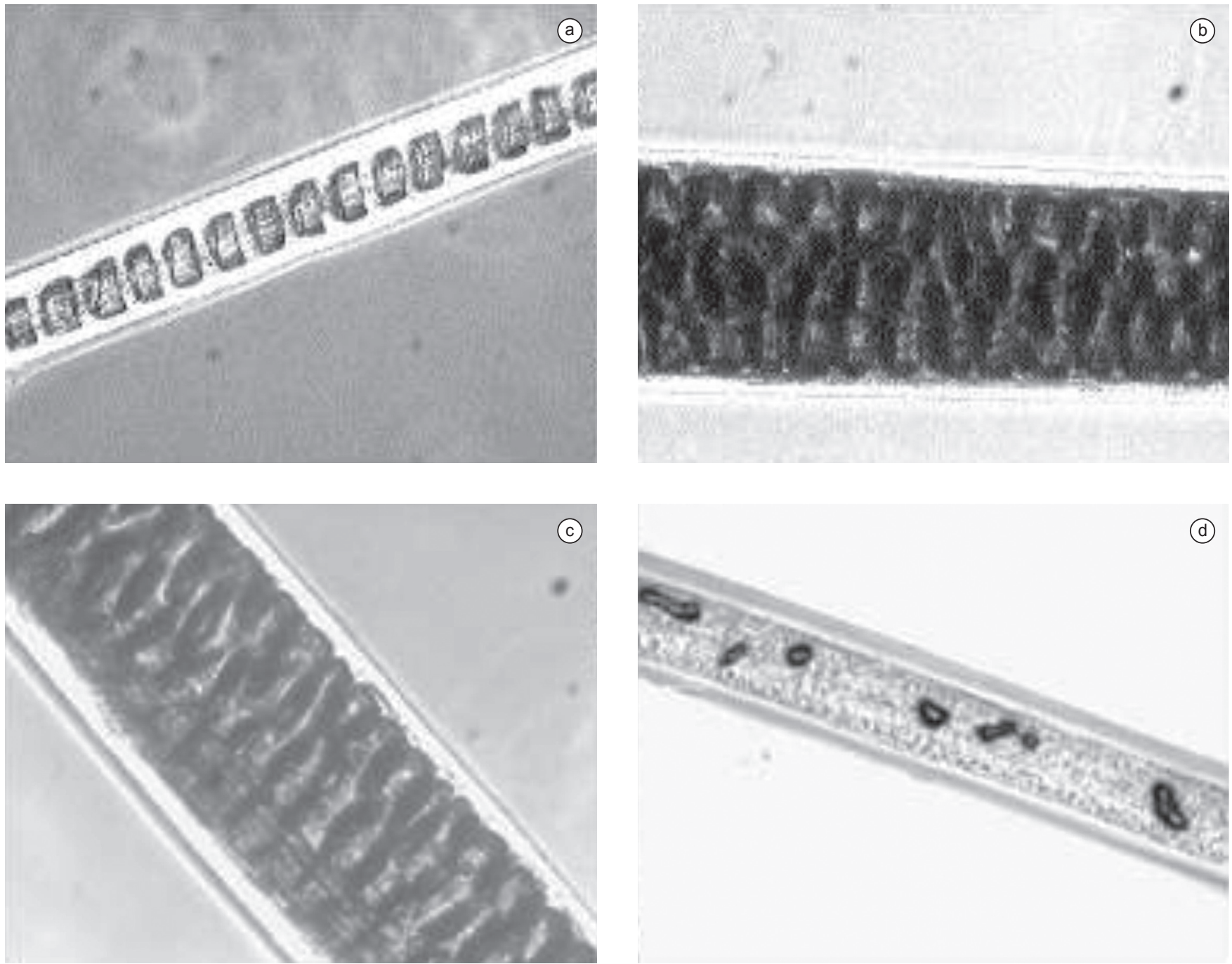

Figura 1. Padrão medular dos pêlos-guardas, em microscopia ótica aumento de 400x, das espécies de pequenos mamíferos não-voadores de agroecossistemas do Estado de São Paulo. a) medula contínua, unisseriada e escalariforme de Gracilinanus agilis; b) medula contínua, multisseriada, com células isoladas em forma de alvéolos de Akodon montensis; c) medula contínua, multisseriada, com células anastomosadas em forma de listras de Oligoryzomys nigripes; e d) medula contínua, multisseriada, com células anastomosadas de forma crivada de Didelphis albiventris.

Figure 1. Medullar pattern of guard hairs in optical microscope amplified 400x of non-volant small mammal from agroecosystems of São Paulo State. a) continuous, uniseriate and scalariform medulla of Gracilinanus agilis; b) continuous, multiseriate medulla with isolated alveoli-shaped cells of Akodon montensis; c) continuous, multiseriate medulla with grafted strip-shaped cells of Oligoryzomys nigripes; and d) continuous, multiseriate medulla with grafted riddle-shaped cells of Didelphis albiventris.

(Figuras 2d, e, f). Em relação à orientação, os padrões identificados foram: a) transversal, com escamas dispostas transversalmente em relação ao eixo longitudinal do pêlo (Figura 2e), b) oblíqua simples, com escamas inclinadas apenas para um lado em relação ao eixo longitudinal do pêlo, com ausência de uma linha central de divergência (Figura 2d) e c) obliqua dupla, quando as escamas dispuseram-se inclinadas para os dois lados opostos, em relação ao eixo longitudinal (Figura 2f). Foram encontradas as seguintes formas de escamas: a) folidácea, onde a superfície do pêlo assemelha-se à pele de uma cobra (Figuras 2b, c e 3a, b, c, d), e b) conoidal, onde cada escama envolve todo o perímetro do pêlo, formando um cone (Figura 2a). As escamas de forma folidácea foram diferenciadas em quatro sub-tipos: a) ovalada, onde o ápice da escama apresenta forma ovalada (Figura 2b, 3a e 3c), b) forma de telhas, onde a superfície do pêlo assemelha-se ao arranjo de telhas em um telhado (Figura 3b), c) alongada, onde as escamas apresentam seu eixo vertical como o mais longo (comprimento), quando comparados com o seu eixo horizontal (largura) (Figura 2c) e d) acuminada, onde o ápice da escama se afunila formando uma ponta (Figura 3d). O padrão cuticular de cada espécie está descrito na Tabela 3.

As 11 espécies analisadas foram classificadas com base nos padrões medular e cuticular de pêlos-guarda, de acordo com a chave dicotômica de 11 etapas (Tabela 4).

\section{Discussão}

A seleção de pêlos-guardas para a análise deve-se às diferenças encontradas entre o padrão medular de um pêlo-guarda e um subpêlo sugeridas por Quadros \& Monteiro-Filho (2006a). Teerink (1991), constatou que na grande maioria dos casos, os subpêlos são inúteis 
Microestrutura de pêlos de pequenos mamíferos

Tabela 2. Padrão medular (continuidade, tipo de fileira, disposição e forma das células) dos pêlos-guardas das espécies de pequenos mamíferos não-voadores de agroecossistemas do Estado de São Paulo.

Table 2. Medullar pattern (continuity, type of row, disposition and shape of cells) of guard hairs of non-volant small mammals from agroecosystems of São Paulo State.

\begin{tabular}{lcccc}
\hline \multicolumn{1}{c}{ Espécie } & Continuidade da medula & Fileira de células & Disposição das células & Forma das células \\
\hline Didelphis albiventris & Contínua & Multisseriada & Anastomosadas & Crivada \\
Monodelphis americana & Contínua & Unisseriada & - & Escalariforme \\
Gracilinanus agilis & Contínua & Unisseriada & - & Escalariforme \\
Gracilinanus microtarsus & Contínua & Unisseriada & - & Escalariforme \\
Calomys tener & Contínua & Multisseriada & Anastomosadas & Alveolar \\
Necromys lasiurus & Contínua & Multisseriada & Anastomosadas & Alveolar \\
Rhipidomys mastacalis & Contínua & Multisseriada & Anastomosadas & Alveolar \\
Bibimys labiosus & Contínua & Multisseriada & Anastomosadas & Alveolar \\
Oligorizomys nigripes & Contínua & Multisseriada & Anastomosadas & Listrada \\
Kannabateomys amblyonyx & Contínua & Multisseriada & Anastomosadas & Crivada \\
Akodon montensis & Contínua & Multisseriada & Isoladas & Alveolar \\
\hline
\end{tabular}
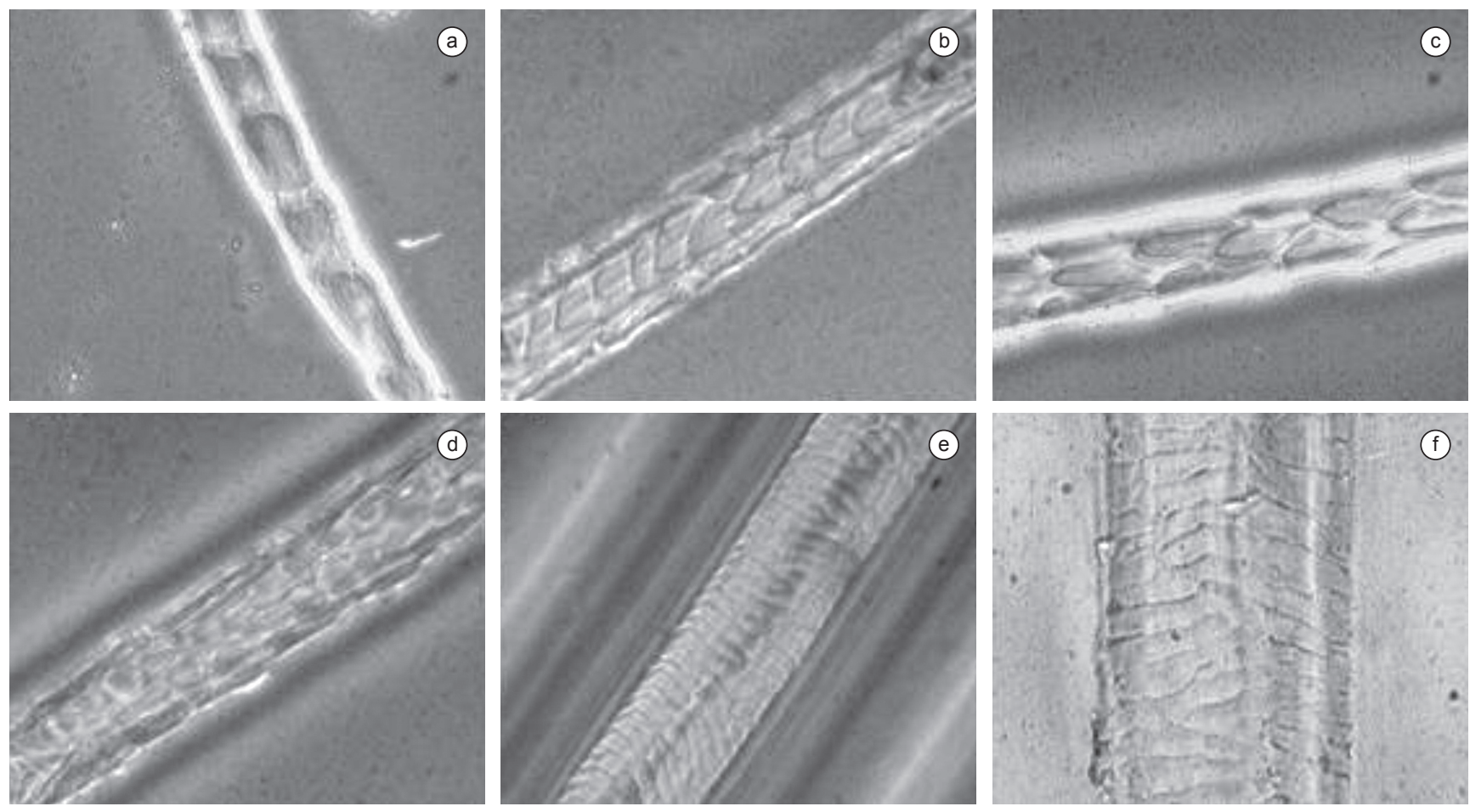

Figura 2. Padrão cuticular dos pêlos-guardas, em microscopia ótica aumento de 400x, das espécies de pequenos mamíferos não-voadores de agroecossistemas do Estado de São Paulo. a) cutícula imbricada com escamas em formato conoidal simples de Gracilinanus agilis; b) cutícula imbricada com escamas em forma de folhas ovaladas de Calomys tener; c) cutícula imbricada com escamas em forma de folhas alongadas de Rhipidomys mastacalis; d) cutícula pavimentosa, com escamas ondeadas, orientadas de forma oblíqua simples e bordas lisas de Necromys lasiurus; e) cutícula pavimentosa, com escamas ondeadas, lisas, orientadas de forma transversal e bordas descontínuas de Didelphis albiventris; e f) cutícula pavimentosa, com escamas ondeadas, orientadas de forma oblíqua dupla de Kannabateomys amblyonyx.

Figure 2. Cuticle pattern of guard hairs in optical microscope amplified 400x of non-volant small mammals from agroecosystems of São Paulo State. a) imbricate cuticle with simple conoidal scales of Gracilinanus agilis; b) imbricate cuticle with oval leaf-shaped scales of Calomys tener; c) imbricate cuticle with long leaf-shaped scales of Rhipidomys mastacalis; d) pared cuticle with simple oblique smoothly bordered wave-shaped scales of Necromys lasiurus; e) pared cuticle with transversal discontinuously bordered wave-shaped scales of Didelphis albiventris, and f) pared cuticle of double oblique wave-shaped scales of Kannabateomys amblyonyx.

na identificação de amostras desconhecidas, pois são semelhantes entre espécies aparentadas.

Dentre os pêlos analisados, a medula apresentou caráter contínuo de acordo com Hausman (1920), Teerink (1991) e Quadros
\& Monteiro-Filho (2006b). Estes mesmos autores apresentam um caráter descontínuo para medula, que não foi detectado neste estudo. Caso fosse possível detectar a descontinuidade da medula, Brunner \& Coman (1974) diferenciam este caráter em dois subtipos "fragmental" 

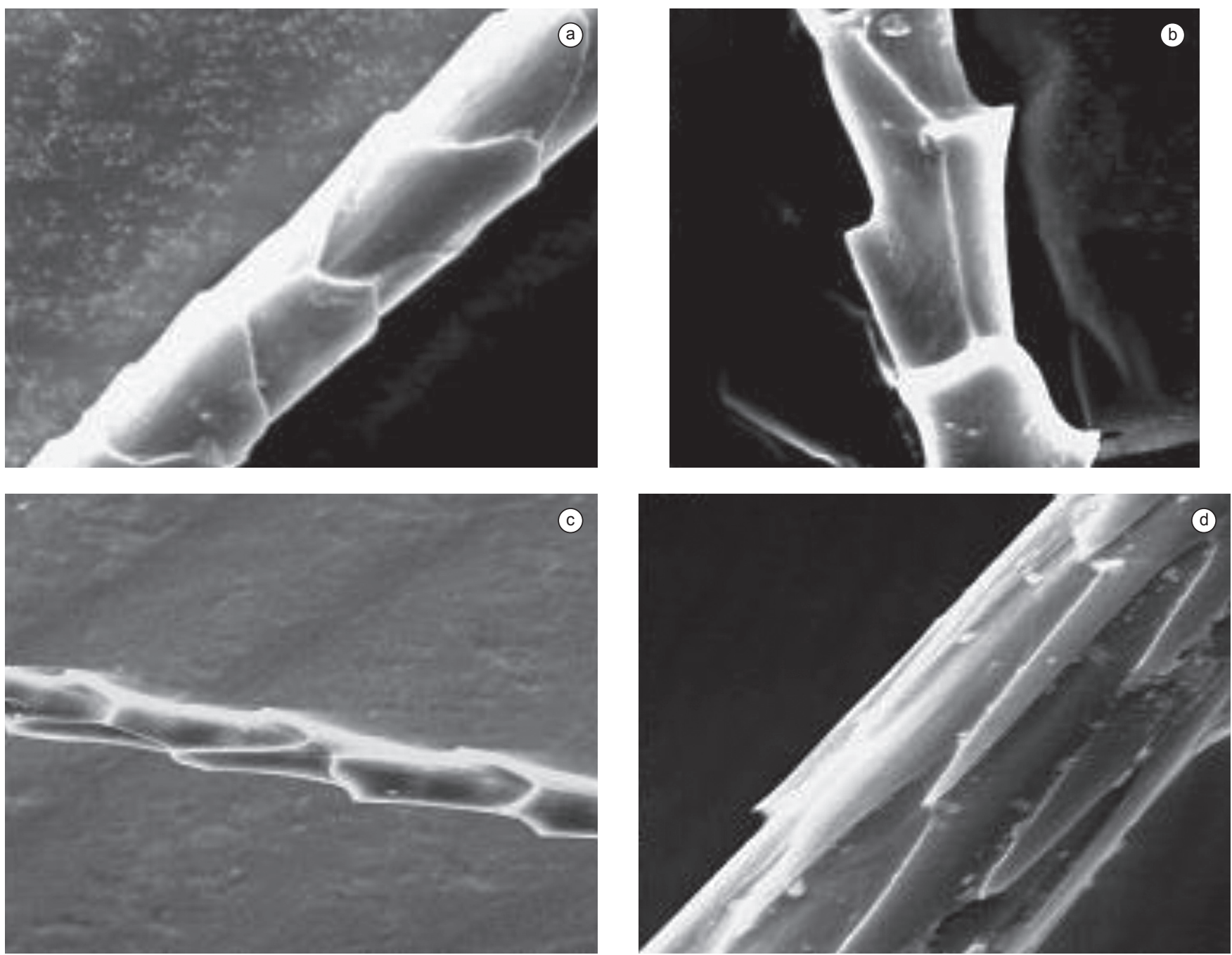

Figura 3. Padrão cuticular dos pêlos-guardas, em microscopia eletrônica de varredura aumento 2000x, das espécies de pequenos mamíferos não-voadores de agroecossistemas do Estado de São Paulo. a) cutícula imbricada com escamas em forma de folhas ovaladas de Monodelphis americana; b) cutícula imbricada, com escamas em formato de telhas de Gracilinanus microtarsus, c) cutícula imbricada com escamas em forma de folhas ovaladas de Calomys tener; e d) cutícula imbricada com escamas em forma de folhas acuminadas de Bibimys labiosus.

Figure 3. Cuticle pattern of guard hairs in electronic microscope amplified 2000x of non-volant small mammal from agroecosystems of São Paulo State. a) imbricate cuticle with oval leaf-shaped scales of Monodelphis americana; b) imbricate cuticle with roof-shaped scales of Gracilinanus microtarsus; c) imbricate cuticle with oval leaf-shaped scales of Calomys tener; and d) imbricate cuticle with acuminate leaf-shaped scales of Bibimys labiosus.

Tabela 3. Padrão cuticular (imbricamento, forma, orientação, ornamentação e continuidade das bordas das escamas) dos pêlos-guardas das espécies de pequenos mamíferos não-voadores de agroecossistemas do Estado de São Paulo.

Table 3. Cuticle pattern (imbrication, shape, orientation, ornamentation and continuity of the border of scales) of guard hairs of non-volant small mammals from agroecosystems of São Paulo State.

\begin{tabular}{|c|c|c|c|c|}
\hline Espécie & Imbricamento & Forma das escamas & $\begin{array}{c}\text { Orientação das } \\
\text { escamas }\end{array}$ & $\begin{array}{c}\text { Ornamentação e continuidade } \\
\text { das bordas das escamas }\end{array}$ \\
\hline Didelphis albiventris & Pavimentosa & Ondeada & Transversal & Lisa e descontínua \\
\hline Monodelphis americana & Imbricada & Folidácea ovalada & - & - \\
\hline Gracilinanus agilis & Imbricada & Conoidal simples & - & - \\
\hline Gracilinanus microtarsus & Imbricada & Folidácea em forma de "telhas" & - & - \\
\hline Calomys tener & Imbricada & Folidácea ovalada & - & - \\
\hline Oligorizomys nigripes & Imbricada & Folidácea alongada & - & - \\
\hline Bibimys labiosus & Imbricada & Folidácea acuminada & - & - \\
\hline Rhipidomys mastacalis & Imbricada & Folidácea alongada & - & - \\
\hline Akodon montensis & Imbricada & Folidácea ovalada & - & - \\
\hline Necromys lasiurus & Pavimentosa & Ondeada & Oblíqua simples & Lisa \\
\hline Kannabateomys amblyonyx & Pavimentosa & Ondeada & Oblíqua dupla & - \\
\hline
\end{tabular}


Tabela 4. Chave dicotômica de identificação de espécies de pequenos mamíferos não-voadores de agroecossistemas do Estado de São Paulo, a partir de características da medula e cutícula de pêlos-guarda.

Table 4. Dichotomous key for the identification of non-volant small mammal species from agroecosystems of São Paulo State, based on medullar and cuticle characteristics of guard hairs.

\begin{tabular}{llc}
\hline 1 & Medula com células unisseriadas escalariformes (Figura 2a) & 2 \\
1 & Medula com células multisseriadas & 4 \\
2 & Cutícula com escamas folídaceas & 3 \\
2 & Cutícula com escamas conoidais (Figura 3a) & Gracilinanus agilis \\
3 & Cutícula com escamas ovaladas (Figura 4a) & Monodelphis americana \\
3 & Cutícula com escamas em forma de telhas (Figura 4b) & Gracilinanus microtarsus \\
4 & Células da medula com disposição isolada (Figura 2b) & Akodon montensis \\
4 & Células da medula com disposição não isolada & 5 \\
5 & Células da medula anastomosadas em forma de alvéolos & 6 \\
5 & Células da medula anastomosadas com formato não alveolar & 7 \\
6 & Cutícula imbricada com escamas folidáceas & 9 \\
6 & Cutícula pavimentosa (Figura 3d) & Necromys lasiurus \\
7 & Células da medula apresentando forma listrada (Figura 2c) & Oligoryzomys nigripes \\
7 & Células da medula apresentando forma crivada (Figura 2d) & 8 \\
8 & Orientação das escamas da cutícula tipo transversal (Figura 3e) \\
8, & Orientação das escamas da cutícula tipo oblíqua dupla (Figura 3f) & Didelphis albiventris \\
9 & Escamas da cutícula em formato ovalado (Figuras 3b e 4c) & Kannabateomys amblyonyx \\
9 & Escamas da cutícula em formato não ovalado & Calomys tener \\
10 & Escamas da cutícula em forma alongada (Figura 3c) & 10 \\
10 & Escamas da cutícula em forma acuminada (Figura 4d) & Rhipidomys mastacalis \\
\hline
\end{tabular}

e "interrupted", de acordo com a quantidade de córtex entre um fragmento e outro de medula.

Hausman (1920), Brunner \& Coman (1974), Teerink (1991) e Quadros \& Monteiro-Filho (2006b), embora tenham atribuído nomes diferentes, dividiram os padrões medulares em duas grandes categorias: medula com uma fileira, ou com duas ou mais fileiras de células. No presente estudo, observou-se esse mesmo padrão e adotou-se essa divisão (medula unisseriada e multisseriada). O padrão medular multisseriado crivado foi descrito pela primeira vez por Quadros \& Monteiro-Filho (2006b) e identificado nas espécies Didelphis albiventris e Kannabateomys amblyonyx do presente estudo.

Para a classificação da cutícula, foi necessária a combinação da nomenclatura e descrição propostas por Hausman (1920) e Quadros \& Monteiro-Filho (2006b), pois o primeiro autor define maiores detalhes em relação ao formato das escamas em seu trabalho. Alguns padrões imbricados da cutícula adotados foram ilustrados por outros autores como sendo pavimentosos (Hausman 1920, Brunner \& Coman 1974, Teerink 1991).

Na análise do imbricamento da cutícula, a técnica de observação através de microscopia óptica demonstrou-se limitada, sendo necessário recorrer à técnica de microscopia eletrônica de varredura. As imagens obtidas pelo microscópio eletrônico de varredura proporcionaram um progresso significativo na descrição destas características bem como no detalhamento do formato e das bordas das escamas, como demonstraram Meng \& Wyss (1997), Quadros \& MonteiroFilho (1998a, b), Chernova (2002) e Meyer et al. (2002). A aplicação da técnica permitiu estabelecer padrões distintos de cutícula para as espécies Monodelphis americana, Gracilinanus microtarsus e Bibimys labiosus. A diferenciação entre M. americana e G. agilis, somente foi possível, analisando todo o comprimento do pêlo. Brunner \& Coman (1974) e Teerink (1991) diferenciaram até quatro tipos de ornamentações das bordas das escamas, que foram úteis na diferenciação entre espécies. Neste estudo, não foi necessária esta diferenciação, pois somente foram verificadas bordas lisas.

Entre os autores que fizeram descrições e sugeriram nomenclaturas para padrões cuticulares apenas Teerink (1991) e Quadros \& Monteiro-Filho (2006b) incluíram a orientação das escamas da cutícula. A interpretação de Teerink (1991) sobre a orientação das escamas em relação ao eixo maior do pêlo confunde-se com as dimensões (largura e comprimento) das escamas. Quando esse autor se refere aos estados de caráter "transversal", "longitudinal" e "intermediate" das escamas cuticulares, está fazendo menção à proporção entre a largura e o comprimento das escamas, o que se reflete na sua orientação em relação ao maior eixo do pêlo. No presente trabalho, por outro lado, os estados de caráter da orientação das escamas (transversal, oblíqua simples e oblíqua dupla) dizem respeito apenas à sua orientação em relação ao eixo longitudinal do pêlo, não tendo relação com as dimensões das escamas e sendo aplicados apenas ao padrão ondeado, como proposto por Quadros \& Monteiro-Filho (2006b).

Os padrões cuticulares e medulares identificados neste trabalho para Oligoryzomys nigripes, D. albiventris, e Necromys lasiurus coincidem com o encontrado por Quadros (2002), já os padrões cuticulares encontrados para M. americana e Gracilinanus microtarsus e medulares para K. amblyonyx diferiram do descrito pela autora. Quadros (2002) descreve a cutícula de M. americana como pavimentosa, sendo neste estudo descrita como imbricada, já no caso da espécie G. microtarsus a autora descreve a forma das escamas da cutícula como conoidal, sendo verificado neste estudo o formato folidáceo. No caso da espécie K. amblyonyx o padrão cuticular coincidiu com o de Quadros (2002), porém o padrão das células da medula de formato crivado, detectado neste estudo, diferiu ao da autora que descreveu o formato destas células como fusiforme. Essas diferenças podem estar 
relacionadas à observação de partes distintas do pêlo, neste estudo a cutícula foi observada na sua região proximal e a medula na sua região distal, que foram as regiões que apresentaram maior variação interespecífica. Quadros (2002), em seu trabalho, não especificou qual região do pêlo apresentava maior variação interespecífica.

Neste estudo, foram estabelecidos padrões para as microestruturas dos pêlos de Akodon montensis, Bibimys labiosus, Gracilinanus agilis, Calomys tener e Rhipidomys mastacalis, espécies para as quais não foram encontrados trabalhos anteriores na literatura. Assim, este estudo contribui para um maior conhecimento sobre a tricologia de mamíferos de pequeno porte não voadores que habitam agroecossistemas do Estado de São Paulo, servindo como subsidio a pesquisas relacionadas à dieta de carnívoros.

\section{Agradecimentos}

Gostaríamos de agradecer aos professores Gilberto J. de Moraes e Elliot W. Kitajima por cederem seus laboratórios para a realização de etapas importantes deste estudo e ao $\mathrm{CNPq}$ pela bolsa concedida.

\section{Referências Bibliográficas}

ANDERSON, M.J. 2001. The use of hair morphology in the classification of Galagos (Primates, Subfamily Galagoninae). Primates, 42(2):113-121.

ANDRUSKIW, M. 2003. Prey abundance, availability, and anxiety in structures environments. University of Guelph, Canada.

BARROS-BATTESTTI, D.M., MARTINS, R., BERTIM, C.R., YOSHINARI, N.H., BONOLDI, V.L.N., LEON, E.P., MIRETZKI, M. \& SCHUMAKER, T.T.S. 2000. Land fauna composition of small mammals of a fragment of Atlantic Forest in the State of São Paulo, Brazil. Rev. bras. zool. 17(1):241-249.

BONVICINO, C.R., LINDBERGH, S.M. \& MAROJA, L.S. 2002. Small nonflying mammals from conserved and altered areas of Atlantic Forest and Cerrado: comments on their potencial use for monitoring environment. Braz. j. biol. 62(4B):765-774.

BRUNNER, H. \& COMAN, B.J. 1974. The identification of mammalian hair. Inkata Press, Melbourne.

BROECK, W.V., MORTIER, P. \& SIMOENS, P. 2000. Scanning electron microscopic study of different hair types in various breeds of rabbits. Folia morphol. 60(1):33-40.

BUSCH, M., MIÑO, M.H., DADON, J.R. \& HODORA, K. 2001. Habitat selection by Akodon azarae and Calomys laucha (Rodentia, Muridae) in pampean agroecosystems. Mammalia, 65(1):29-48.

CASTRO, E.B.V. \& FERNANDEZ, F.A.S. 2004. Determinants of differential extinction vulnerabilities of small mammals in Atlantic forest fragments in Brazil. Biol. conserv. 119(1):73-80.

CARTER, B.C. \& DILWORTH, T.G. 1971. A simple technique for revealing the Surface Pattern of Hair. Am. midl. nat. 85(1):260-262.

CHEHÉBAR, C. \& MARTíN, S. 1989. Guía para el reconocimiento microscópico de los pelos de los mamíferos de la Patagonia. Donana. Acta vertebr. 16(2):247-291.

CHERNOVA, O.F. 2002. Architectonic and diagnostic significance of hair cuticle. Biology Bulletin, 29(3):238-247. CYPHER, B.L. 1993a. Food item use by coyote pups at crab orchard national wildlife refuge, Illinois. Transactions Illinois State Academy Science, 86(3-4):133-137.

CYPHER, B.L., WOOLF, A. \& YANCY, D.C. 1993b. Summer food habits of coyotes at Union County Conservation Area, Illinois. Transactions Illinois State Academy Science, 86(3-4):145-152.

FERNÁNDEZ, G.J. \& ROSSI, S.M. 1998. Medullar type and cuticular scale patterns of hairs of rodents and small marsupials from the Monte Scrubland (San Luis Province, Argentina). Mastozool. neotrop. 5(2):109-116

FONSECA, G.A.B. \& ROBINSON, J.G. 1990. Forest size and structure: competitive and predatory effects on small mammal communities. Biol. conserv. 53(4):265-294.
GHELER-COSTA, C., VERDADE, L.M. \& ALMEIDA, A.F., 2002. Mamíferos não-voadores do campus "Luiz de Queiroz”, Universidade de São Paulo, Piracicaba, Brasil. Rev. bras. zool. 19(2):203-214.

GHELER-COSTA, C. 2006. Distribuição e abundância de pequenos mamíferos em relação à paisagem da bacia do Rio Passa-Cinco, São Paulo, Brasil. Tese de Doutorado, Universidade de São Paulo, Piracicaba.

GONZÁLEZ-ESTEBAN, J., VILLATE, I. \& IRIZAR, I. 2006. Differentiating hair samples of the European mink (Mustela lutreola), the American mink (Mustela vison) and the European polecat (Mustela putorius) using light microscopy. J. zool. 270(3):458-461.

HAUSMAN, L.A. 1920. Structural characteristics of the hair mammals. Am. Nat. 54(635):496-523.

HILTON, H.J. \& KUTSCHA, N.P. 1978. Distinguishing characteristics of the hairs of eastern coyote, domestic dog, red fox and bobcat in Maine. Am. midl. nat. 100(1):223-227.

HOEY, K.A., WISE, R.R \& ADLER, G.H. 2004. Ultrastructure of echimyid and murid rodent spines. J. zool. 263(3):307-315.

HOUCK, M.M. 2002. Hair bibliography for the forensic scientist. Forensic Scientist Communications (40): http://www.fbi.gov/hq/lab/fsc/backissu/ jan2002/houck.htm (último acesso em 01/04/2009).

INGBERMAN, B. \& MONTEIRO-FILHO, E.L.A. 2006. Identificação microscópica dos pêlos das espécies brasileiras de Alouatta Lacépéde, 1799 (Primates, Atelidade Alouattinae). Arquivos Museu Nacional, 64(1):61-71.

KELLER, A. 1980. Détermination des mammifères de la Suisse par leus pelage: II: Diagnose des famillas III. Lagormorpha, Rodentia (partim). Revue Suisse Zoology, 87(3):781-796.

KITAJIMA, E.W. \& LEITE, B. 1999. Curso introdutório de microscopia eletrônica de Varredura. 2 ed. ESALQ; USP, Piracicaba, São Paulo.

KOFFLER, N.F. 1993. Uso das terras da bacia do rio Corumbataí em 1990. Geografia, 18(1):135-150.

MARINIS. A.M. \& ASPREA, A. 2006. How did domestication change the hair morphology in sheep and goats?. Hum. evol. 21(2):139-149.

MATHIAK, H.A. 1938. A key to hairs of the mammals of southern Michigan. J. wildl. manage. 2(4):251-268.

MAYER, W.V. 1952. The hair of California mammals with keys to the dorsal guard hairs of California mammals. Am. midl. nat. 48(2):480-512.

MENG, J. \& WISS, A.R. 1997. Multituberculate and other mammal hair recovered from palaeogene excreta. Nature, 385(6618):712-714.

MEYER, W., SCHNAPPER, A. \& HÜLMANN, G. 2002. The hair cuticle of mammals and its relationship to functions of the hair coat. J. zool. 256(4):489-494

MEYER, W., SCHNAPPER, A., HÜLMANN, G. \& SEGER, H. 2000. Domestication-related variations of the hair cuticula pattern in mammals. J. anim. breed. genet. 117(4):281-283.

MÜLLER, M.V.Y. 1989. Microestrutura de pêlos de mamíferos: métodos de análise e sua plicação na identificação de algumas espécies do estado do Paraná, Brasil. Dissertação de Mestrado, Universidade Federal do Paraná, Curitiba.

NASON, E.S. 1948. Morphology of hair of Eastern North American bats. Am. midl. nat. 39(2):345-361.

ODUM, H.T., ODUM, E.C., BROWN, M.T., LAHART, D., BERSOK, C., SENDZIMIR, J., SCOTT, G.B., SCIENCEMAN, D. \& MEITH, N. 1988. Environmental systems and public policy. University of Florida, Gainesville.

PARDINI, R. 2004. Effects of forest fragmentation on small mammals in an Atlantic Forest landscape. Biodiversity and conservation, 13(13):25672586.

PARDINI, R., SOUZA, M.S., BRAGA-NETO, R. \& METZGER, J.P. 2005. The role of forest structure, fragment size and corridors in maintaining small mammal abundance and diversity in an Atlantic Forest landscape. Biol. Conserv. 124(2):253-266. 
PIANTANIDA, M. \& PETRIELLA, A.M. 1976. Estudio morfológico de algunas especies de roedores de la Provincia de Buenos Aires con el microscopio electrónico de barrido. Physis, 35(90):105-124.

PIRES, A.S., LIRA, P.K., FERNANDEZ, F.A.S., SCHITTINI, G.M. \& OLIVEIRA, L.C. 2002. Frequency of moviments of small mammals among Atlantic Coastal Forest fragments in Brazil. Biol. Conserv. 108(2):229-237.

PIRES, A.S., FERNANDEZ, F.A.S., FREITAS, D. \& FELICIANO, B.R 2005. Influence of edge and fire-induced changes on spacial distribution of small mammals in Brazilian Atlantic Forest fragments. Studies on Neotropical Fauna and Environment 40(1):7-14.

PORTA, X. \& LLANEZA, L. 2001. Diferencias macroscópicas entre pelos de perro (Canis familiaris Linnaeus, 1758) y lobo ibérico (Canis lupus signatus Cabrera, 1907). Galemys, 13(no. especial):205-215.

QUADROS, J. 1998. Identificação microscópica de pelos de mamíferos brasileiros e sua aplicação no estudo de dieta de carnívoros. Tese de Doutorado, Universidade Federal do Paraná, Curitiba.

QUADROS, J. \& MONTEIRO-FILHO, E.L.A. 1998a. Effects of digestion, putrefaction, and taxidermy process on Didelphis albiventris hair morphology. J. zool. 244(3):331-334.

QUADROS, J. \& MONTEIRO-FILHO, E.L.A. 1998b. Morphology of different hair types of Didelphis albiventris and its usage in hair identification. Cien. cult. 50(5):382-385.
QUADROS, J. \& MONTEIRO-FILHO, E.L.A. 2006a. Coleta e preparação de pêlos de mamíferos para identificação em microscopia óptica. Rev. bras. zool. 23(1):274-278.

QUADROS, J. \& MONTEIRO-FILHO, E.L.A. 2006b. Revisão conceitual, padrões microestruturais e proposta nomenclatória para os pêlos-guarda de mamíferos brasileiros. Rev. bras. zool. 23(1):279-296.

TEERINK, B.J. 1991. Hair of west European mammals: atlas and identification. Cambridge University Press, Cambridge.

VALENTE, R.O. A. 2001. Análise da estrutura da paisagem na bacia do rio Corumbataí. Dissertação de Mestrado, Universidade de São Paulo, Piracicaba.

VAZQUEZ, D.E., PEROVIC, P.G. \& OLSEN, A.A. 2000. Patrones cuticulares y medulares de pelos de mamíferos del noroeste argentino (Carnivora y Artiodactyla). Mastozool. neotrop. 7(2):131-142.

WEINGART, E.L. 1973. A simple technique for revealing hair scale patters. Am. midl. nat. 90(2):508-509.

Recebido em 06/02/08 Versão reformulada recebida em 02/12/08 Publicado em 03/02/09 
